\title{
Desmoplastic Nevus
}

National Cancer Institute

\section{Source}

National Cancer Institute. Desmoplastic Nevus. NCI Thesaurus. Code C4497.

A benign melanocytic nevus characterized by the presence of desmoplastic stroma. 